\title{
Impact of Airflow Communication between Nasal Cavities on Nasal Ventilation
}

\author{
Bing Zhou ${ }^{a}$ Qian Huang ${ }^{a}$ Shunjiu Cui ${ }^{a}$ Yingxi Liu ${ }^{b}$ Demin Han ${ }^{a}$ \\ a Department of Otolaryngology, Head and Neck Surgery, State Key Laboratory of \\ Otolaryngology, Head and Neck Surgery, Ministry of Education, Beijing Tongren Hospital, \\ Capital Medical University, Beijing, and bepartment of Engineering Mechanics, \\ State Key Laboratory of Structural Analysis for Industrial Equipment, Dalian University of \\ Technology, Dalian, China
}

\section{Key Words}

Nasal septal deviation · Chronic rhinosinusitis · Aerodynamics · Correlation · Computational fluid dynamics

\begin{abstract}
Objective: To investigate the impact of airflow communication between bilateral nostril sides on nasal ventilation. In addition, we try to validate the efficacy of the Draf III procedure from the aerodynamics perspective. Methods: One health model and two disease models were constructed. These included 2 patients with nasal septum perforation and 1 patient who received the Draf III procedure. With the computational fluid dynamics method, indices such as airflow velocity and wall shear stress in the nasal cavity were detected and compared among the 3 subjects. Results: The main pathway for airflow in the nasal cavity is the common meatus. Little airflow exchange occurred in the patient who underwent the Draf III procedure, and the wall shear stress around the communication site was as low as in the adjacent areas. However, when airflow communication occurred in the lower part of the nasal cavity, the airflow velocity and wall shear stress were obviously altered, and the ventilation function of the nasal cavity was impaired. Conclusion: Airflow communication in the upper part of the nasal cavity has little impact on nasal ventilation. Nonetheless, airflow communication occurring in the lower part of the nasal cavity disturbs the overall airflow distribution and a repair procedure is necessary.




\section{Introduction}

Airflow communication between bilateral nostril sides is a common entity in rhinological procedures. Nasal septal perforation is one of the most common manifestations, which causes symptoms such as crusting, drying, epistaxis, whistling and nasal obstruction $[1,2]$. The consequences are devastating and a repair procedure is always necessary.

Several studies on the airflow pattern in patients with nasal septal perforation have been described. Nasal septal perforation disturbs the airflow pattern, which impairs the humidification and warming function of the nasal cavity [3-5]. The high wall shear stress is always located in the posterior region of perforation, which could explain drying and epistaxis. Several perforation models with different sizes and locations have been investigated, and it has been confirmed that the devastating symptoms are correlated with the size of perforation but not with location [6-8].

However, there is another category of airflow communication in rhinological procedures, which was observed in patients with recalcitrant frontal diseases needing a Draf III procedure $[9,10]$. It mainly manifests as a new window creation around the frontal recess. Surgical dissection would induce airflow communication between bilateral nostril sides. In the longterm follow-up, there were no such devastating symptoms in patients who received the Draf III procedure as in patients with nasal septum perforation [10].

What is the cause of this difference? The main difference between patients with nasal septal perforation and patients who received the Draf III procedure was that one communication occurs in the lower part of the nasal cavity and the other one in the upper part. However, the locations in previous researches were limited to the anteroposterior direction, not considering that the airflow communication occurred in the upper and lower part of the nasal cavity. Furthermore, no research related to airflow field alterations after the Draf III procedure has been reported so far.

We adopted the computational fluid dynamics (CFD) method [11-16] to simulate the airflow characteristics of the nasal cavity in different situations. By virtue of visualization, the indices of velocity, pressure, wall shear stress, velocity vector and streamlines can be detected accurately.

The purpose of our study was to explore the impact of airflow communication on nasal ventilation in the upper and lower part of the nasal cavity. Moreover, we tried to validate the efficacy of the Draf III procedure from the airflow dynamics perspective.

\section{Materials and Methods}

A total of 3 subjects, 1 healthy test person, 1 patient with nasal septum perforation (with symptoms of nasal obstruction, crusting and epistaxis) and 1 patient who had undergone Draf III surgery (with no symptoms), underwent nasal computed tomography (CT) scans to construct the 3D model. All subjects were informed of the flaws of the CT scan. Nasal CT scans, combining the axial, coronal and sagittal directions, were implemented.

DICOM (Digital Imaging and Communications in Medicine) format images were obtained at a spatial resolution of $512 \times 512$ pixels and $0.625 \mathrm{~mm}$ thickness. Segmentation and primary smoothing of the raw images were performed using Mimics 13.1. In addition, the perforation sizes were also measured using Mimics 13.1, and the sizes for septal perforation and Draf III surgery were $10.49 \times 11.09$ and $12.32 \times 16.71$ $\mathrm{mm}^{2}$, respectively. The Mimics models were then imported into Geomagic studio 12 (Geomagic, N.C., USA) for further smoothing and surface partition, followed by acquisition of surface elements of the IGES (Initial Graphics Exchange Specification) format for meshing.

The meshing work was implemented in ICEM-CFD (The Integrated Computer Engineering and Manufacturing code for Computational Fluid Dynamics; ANSYS, Canonsburg, Pa., USA), and global and local controls were taken to improve mesh quality. Then, the meshes with a maximal skewness value of less than 0.6 were 
Fig. 1. Coronal sections of 2 patients are shown. a CT scan of the patient with septum perforation. b CT scan of the patient who underwent the Draf III procedure.

Zhou et al.: Impact of Airflow Communication between Nasal Cavities on Nasal Ventilation

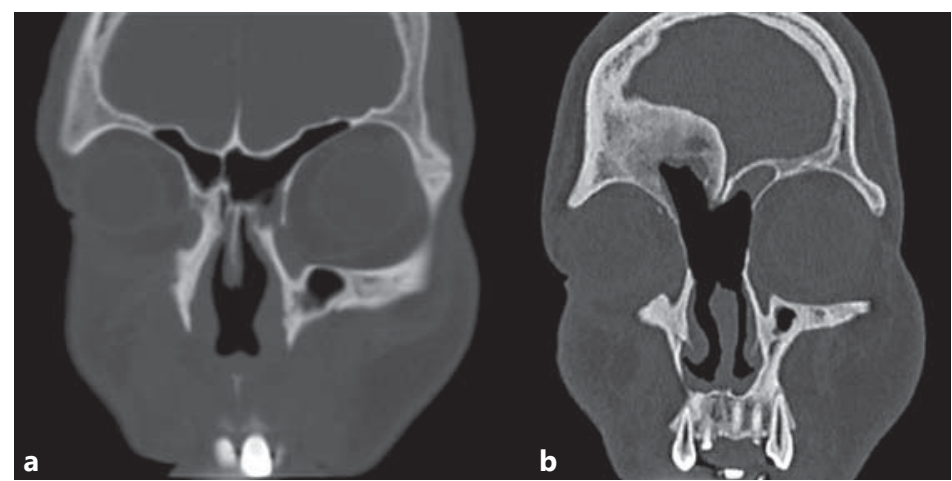

imported into Fluent 13.0 (ANSYS) for solution procedures. To improve the accuracy of numerical simulation, mesh-independent analysis was conducted in each individual to select the appropriate mesh number for the final analysis.

In CFD simulations, the flow was assumed to be incompressible and quasi-steady. Characteristic values for airflow and temperature fields were derived from the continuity, Navier-Stokes and energy equations. The RNG (Renormalization Group) k-epsilon turbulence model was chosen for CFD simulation. The SIMPLEC (Semi-Implicit Method for Pressure-Linked Equations Consistent) algorithm and second-order upwind format discretization of the momentum, turbulent kinetic energy, turbulent dissipation rate and energy were adopted.

Atmospheric pressure was imposed on both nostrils. The nasal wall was assumed to be no-slip $(\mathrm{u}=\mathrm{v}=$ $0)$. A constant airflow rate of $36 \mathrm{l} / \mathrm{min}$ was applied to the pharynx boundary.

Data processing was conducted using CFD-Post (ANSYS) to obtain contour visualization and numerical outputs. We additionally created a coronal plane crossing the communication in 3D models to visualize airflow velocity and wall shear stress. The indices of maximal and average airflow velocity, and maximal and average wall shear stress crossing the communication were analyzed and compared between the 3 subjects.

\section{Results}

In the patient with nasal septal perforation, the communication site occurred in the lower part of the nasal cavity (fig. 1a). However, the communication site in the patient who underwent the Draf III procedure was located in the upper part of the nasal cavity (fig. 1b).

Compared to the healthy subject (fig. 2a), the patient who underwent the Draf III procedure showed the same characteristics, namely that the airflow was mainly distributed in the common meatus (fig. 2b) and that the airflow exchange in the communication site was little. The main airflow in the patient with nasal septal perforation was also distributed in the common meatus. Nevertheless, there was much more airflow exchange in the communication site (fig. 2c, d).

The maximal airflow velocity in the patient with nasal septal perforation was $14.75 \mathrm{~m} / \mathrm{s}$ and was located in the nasal valve area, while in the healthy subject and in the patient who underwent the Draf III procedure, the maximal velocities were 10.55 and $9.59 \mathrm{~m} / \mathrm{s}$, respectively, both of which were located in the nasal valve area. To explore the alteration caused by airflow communication, we created a coronal plane crossing the communication site. The average velocity in the patient with septum perforation was $2.64 \mathrm{~m} / \mathrm{s}$ (fig. 3a), while this value in the Draf III procedure patient was $1.05 \mathrm{~m} / \mathrm{s}$ (fig. $3 \mathrm{~b}$ ).

The maximal wall shear stress in the patient with nasal septal perforation was $3.31 \mathrm{~Pa}$ and was located in the nasal valve area. The wall shear stress around the communication site 
Zhou et al.: Impact of Airflow Communication between Nasal Cavities on Nasal Ventilation

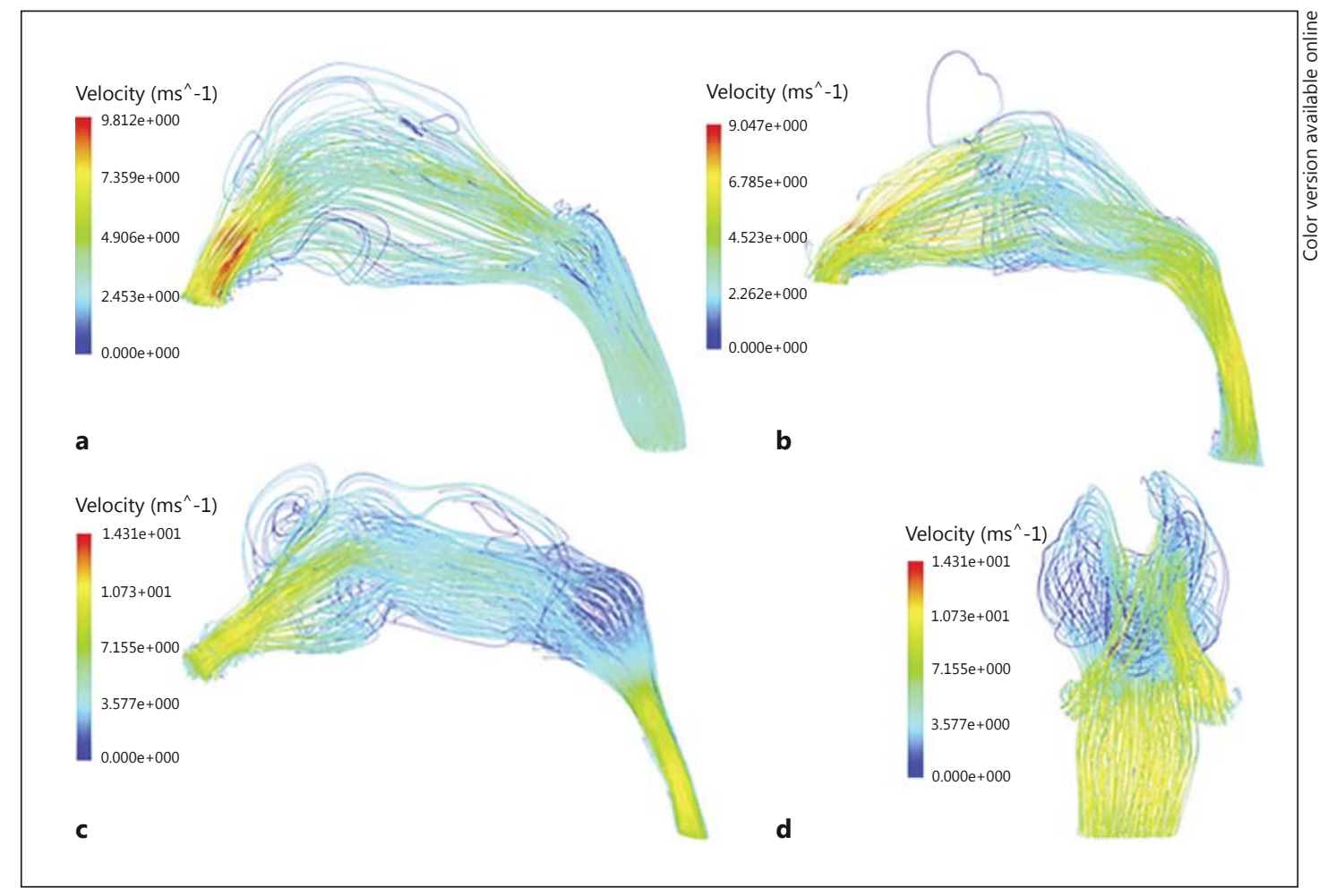

Fig. 2. Streamline distribution in 3 cases. a Healthy subject. b Patient who underwent the Draf III procedure. Patient with septum perforation: the lateral view is shown in $\mathbf{c}$ and the anterior view is shown in $\mathbf{d}$.

Fig. 3. Velocity contours. a Coronal section crossing the communication site in the patient with septum perforation. b Coronal section crossing the communication site in the patient who underwent the Draf III procedure.

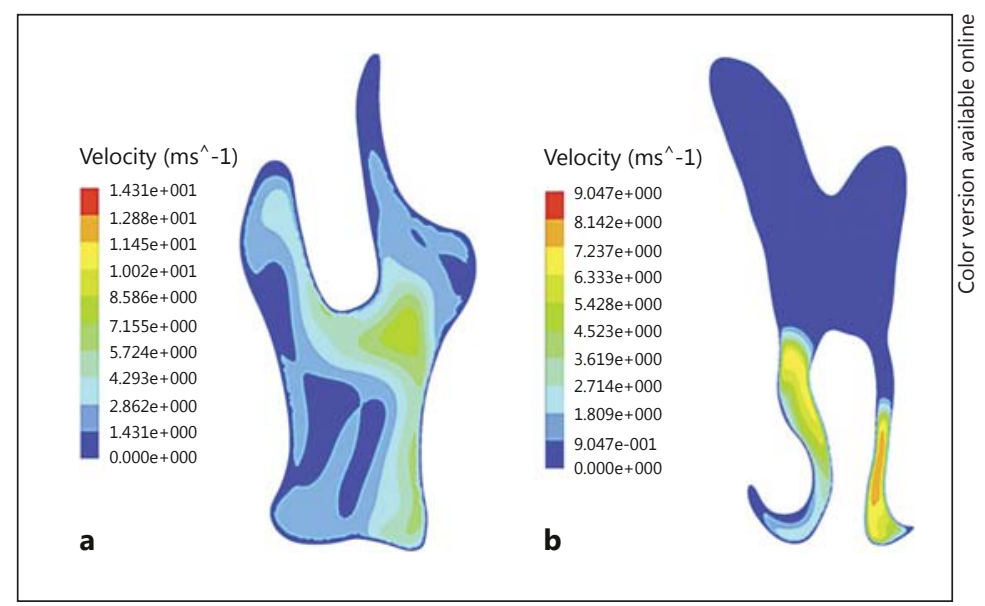

was much higher than in the adjacent area (fig. 4a, b), which was in accordance with researches from Pless et al. [4], Lee et al. [6] and Grant et al. [8]. The increase of the wall shear stress around the communication site may cause symptoms such as drying and epistaxis. However, in the Draf III procedure patient, the maximal wall shear stress was $1.98 \mathrm{~Pa}$ and was located in the nasal valve area. The wall shear stress around the communication site was as low as in other parts of the nasal cavity (fig. 4c, d). Although significant structure alterations in the Draf III procedure patient occurred, no significant change in the mucosal wall shear stress could 
Zhou et al.: Impact of Airflow Communication between Nasal Cavities on Nasal Ventilation

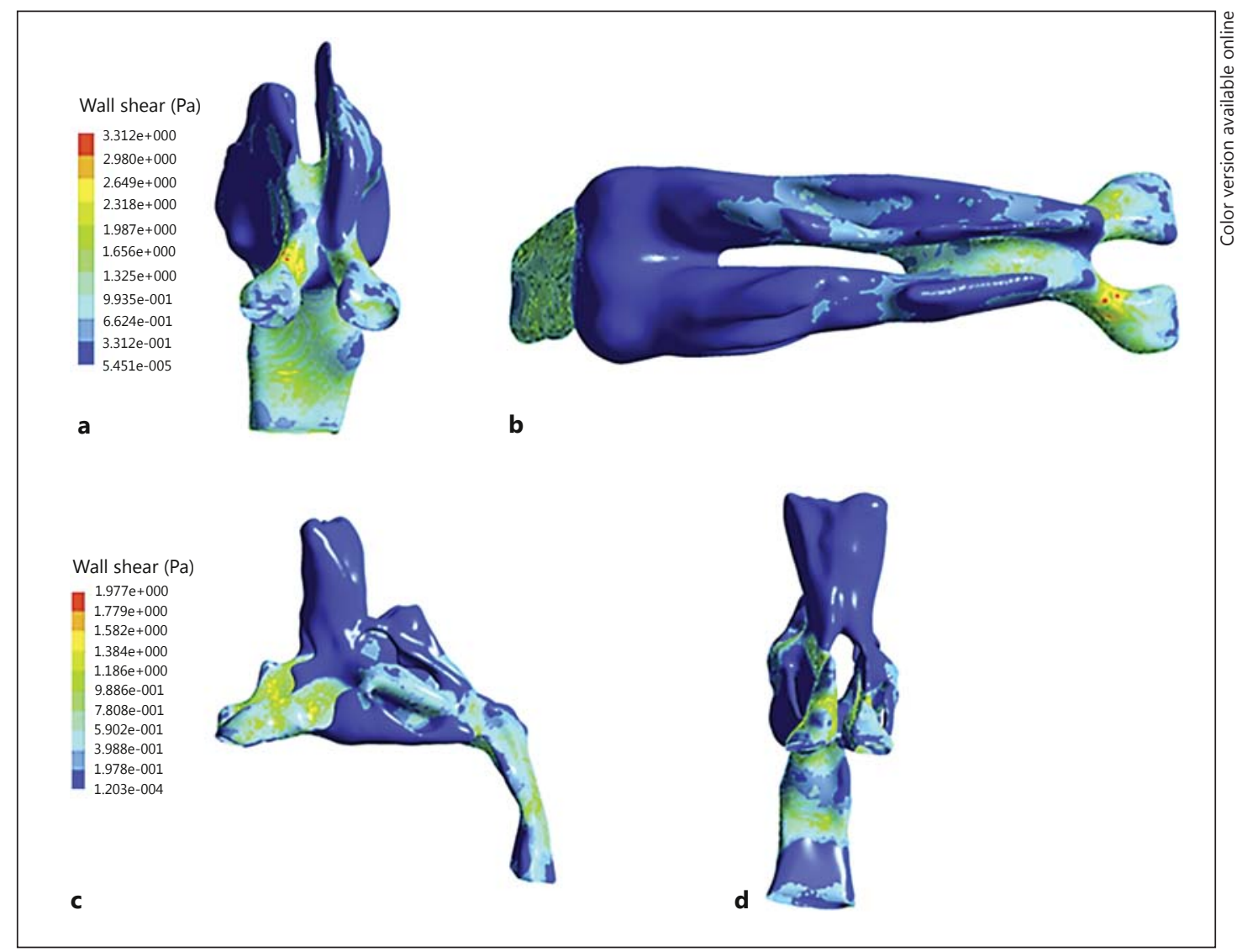

Fig. 4. Wall shear stress contours. Patient with septum perforation: the anterior view is shown in a and the upper view is shown in b. Patient who underwent the Draf III procedure: the lateral view is shown in $\mathbf{c}$ and the anterior view is shown in $\mathbf{d}$.

be observed. This could be an explanation for the occurrence of silent symptoms after the Draf III procedure.

A coronal plane crossing the communication site was created to detect the velocity vector, which revealed the route of airflow movement. We could observe that the cross-action between bilateral nostril sides in the patient with nasal septal perforation (fig. 5a) was much higher than that in the Draf III procedure patient (fig. 5b), similar to the magnitude of velocity in the interchange part.

\section{Discussion}

The purpose of this study was to investigate the impact of airflow communication in the upper and lower part of the nasal cavities on nasal functions. We could observe that airflow communication occurring in the upper part of the nasal cavity had little impact on airflow distribution. However, airflow communication in the lower part of the nasal cavity had great impact on airflow allocation, which was concretely shown by the indices of the main airflow route, velocity and wall shear stress.

In the healthy subject, the main passage for airflow was the common meatus, which is in accordance with Leong et al. [17]. Moreover, the airflow was mainly located in the lower two 
Fig. 5. Vector contours. a Coronal section crossing the communication site in the patient with septum perforation. b Coronal section crossing the communication site in the patient who underwent the Draf III procedure.

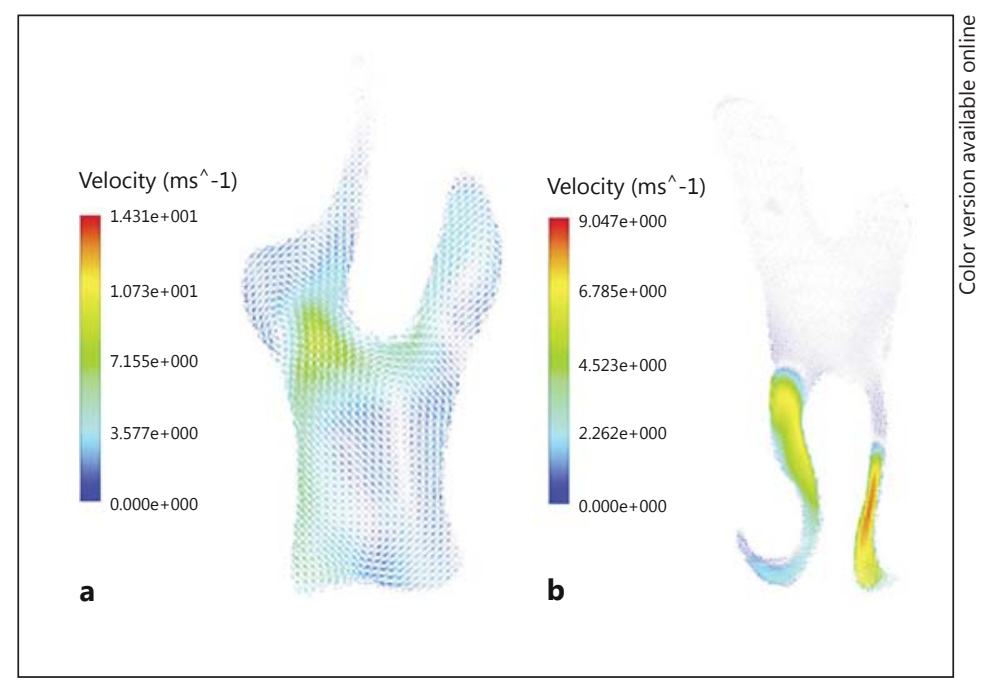

thirds of the common meatus. Due to the small airflow volume, the airflow communication occurring in the upper part of the nasal cavity had little impact on the airflow pattern in the Draf III procedure patient. However, the communication site where the septum perforation was located represented the main airflow passage in the nasal cavity. The disturbance originating from a structural abnormality will definitely be detrimental to nasal ventilation. Thus, the consequences caused by the septum perforation were devastating [2].

The wall shear stress in the posterior part of the perforation was higher than in the anterior part, which is in accordance with the results of Lee et al. [6] and Grant et al. [8]. Compared to the healthy subject, the overall velocity magnitude and maximal velocity increased in the patient with nasal septal perforation. In the patient with nasal septal perforation near the communication area, the wall shear stress was also much higher than in the adjacent area. The accelerated airflow would reduce the contact time of airflow on the mucosa and increased wall shear stress would augment the stimulation of airflow to the nasal mucosa, both of which would damage the physiological function of the nasal cavity. Both Lee et al. [6] and Grutzenmacher et al. [7] considered that the impact of airflow communication in septum perforation correlated with the size but not the location of perforation. However, their researches were limited to the anteroposterior direction. In our study, the difference caused by airflow communication in the upper and lower part of the nasal cavity was described at length.

The maximal wall shear stress in the Draf III procedure patient was $1.98 \mathrm{~Pa}$ and was located in the nasal valve area. Moreover, the wall shear stress around the communication site was as low as in the adjacent area. The average velocity in the coronal plane crossing the communication site was $1.05 \mathrm{~m} / \mathrm{s}$. The lower the airflow velocity and wall shear stress, the less the stimulus of airflow that was brought to the nasal mucosa. Considering alterations of velocity and wall shear stress, Draf III procedure patients almost had the same characteristics as healthy subjects, which may be the cause of the silent symptoms in Draf III procedure patients in the long-term follow-up.

Although airflow communication occurred, the main airflow passage was still located in the common meatus and the key constrictive area still lied in the nasal valve area in our 3 subjects. However, the airflow velocity and wall shear stress distribution were completely different. Here, the only interpretation is that one communication was located in the lower part of the nasal cavity and the other one in the upper part. The consequences were devastating and showed great correlation with the communication sites. 
Furthermore, the Draf III procedure is becoming a popular surgical technique in the treatment of recalcitrant frontal diseases [18]. Through the surgery, the communication between the bilateral nasal cavities is restored; however, whether the ventilation function of the nasal cavity will be impaired needs to be investigated in future studies. We could confirm that the Draf III procedure leads to little impairment in the whole airflow pattern. To our knowledge, this is the first report on airflow characteristics in a patient who has undergone a Draf III procedure.

There are several limitations to our study. Firstly, the sample used for analysis was small, thus, a larger sample size of patients who have undergone a Draf III procedure is needed to provide practical suggestions for rhinological surgeons. Secondly, the perforation size was not emphasized in this study; therefore, future studies combining various communication sizes and sites still need to be conducted. Thirdly, the results were detected from computational simulation, and there were no physical experiments for validation. Furthermore, a validation experiment should also be performed in the future.

\section{Conclusion}

Airflow communication in the upper part of the nasal cavity after a Draf III procedure has little impact on nasal ventilation. However, airflow communication in the lower part of the nasal cavity obviously disturbs the airflow distribution and a repair procedure is necessary.

\section{Acknowledgement}

This work was supported by the Beijing Natural Science Foundation of China (Grant No. 7100002) and the Support Funding for Training High-Level Health Technicians of Beijing Health System (No. 2009-3-36).

\section{References}

1 Wong S, Raghavan U: Outcome of surgical closure of nasal septal perforation. J Laryngol Otol 2010;124:868874.

2 Mansour HA: Repair of nasal septal perforation using inferior turbinate graft. J Laryngol Otol 2011;125:474478.

- 3 Li F, Liu Q, Yu H, Zhang Z: Pedicled local mucosal flap and autogenous graft for the closure of nasoseptal perforations. Acta Otolaryngol 2011;131:983-988.

- 4 Pless D, Keck T, Wiesmiller KM, Lamche R, Aschoff AJ, Lindemann J: Numerical simulation of airflow and temperature during inspiration in a nose model with septal perforation. International Congress Series 2004; 1268:1304.

- 5 Lee JY, Lee SH, Kim SC, Koh YW, Lee SW: Usefulness of autologous cartilage and fibrin glue for the prevention of septal perforation during septal surgery: a preliminary report. Laryngoscope 2006;116:934-937.

- 6 Lee HP, Garlapati RR, Chong VF, Wang DY: Effects of septal perforation on nasal airflow: computer simulation study. J Laryngol Otol 2010;124:48-54.

7 Grutzenmacher S, Mlynski R, Lang C, Scholz S, Saadi R, Mlynski G: The nasal airflow in noses with septal perforation: a model study. ORL J Otorhinolaryngol Relat Spec 2005;67:142-147.

8 Grant O, Bailie N, Watterson J, Cole J, Gallagher G, Hanna B: Numerical model of a nasal septal perforation. Stud Health Technol Inform 2004;107:1352-1356.

- 9 Gotlib T, Niemczyk K, Balcerzak J, Krzeski A, Held-Ziolkowska M: Draf III procedures: the ENT Department, Medical University of Warsaw experience (in Polish). Otolaryngol Pol 2010;64:40-43.

10 Georgalas C, Hansen F, Videler WJ, Fokkens WJ: Long terms results of Draf type III (modified endoscopic Lothrop) frontal sinus drainage procedure in 122 patients: a single centre experience. Rhinology 2011;49: 195-201.

11 Lindemann J, Keck T, Wiesmiller K, Sander B, Brambs HJ, Rettinger G, Pless D: Nasal air temperature and airflow during respiration in numerical simulation based on multislice computed tomography scan. Am J Rhinol 2006;20:219-223. 
12 Chen XB, Lee HP, Chong VF, Wang de Y: Assessment of septal deviation effects on nasal air flow: a computational fluid dynamics model. Laryngoscope 2009;119:1730-1736.

13 Zhu JH, Lee HP, Lim KM, Lee SJ, Wang DY: Evaluation and comparison of nasal airway flow patterns among three subjects from Caucasian, Chinese and Indian ethnic groups using computational fluid dynamics simulation. Respir Physiol Neurobiol 2011;175:62-69.

14 Rhee JS, Pawar SS, Garcia GJ, Kimbell JS: Toward personalized nasal surgery using computational fluid dynamics. Arch Facial Plast Surg 2011;13:305-310.

15 Lee JH, Na Y, Kim SK, Chung SK: Unsteady flow characteristics through a human nasal airway. Respir Physiol Neurobiol 2010;172:136-146.

16 Huang Y, White DP, Malhotra A: Use of computational modeling to predict responses to upper airway surgery in obstructive sleep apnea. Laryngoscope 2007;117:648-653.

17 Leong SC, Chen XB, Lee HP, Wang DY: A review of the implications of computational fluid dynamic studies on nasal airflow and physiology. Rhinology 2010;48:139-145.

-18 Silverman JB, Prasittivatechakool K, Busaba NY: An evidence-based review of endoscopic frontal sinus surgery. Am J Rhinol Allergy 2009;23:e59-e62. 